\title{
Agenda Redes
}

\section{Unión Latinoamericana de \\ Extensión Universitaria (ULEU)}

- Red de Observatorios y Cátedras Abiertas y/o Libres de Extensión Universitaria: esta iniciativa está impulsada por la Secretaría de Extensión de UNL.

-X Congreso Internacional de Educación Superior "Universidad 2016" bajo el lema "Universidad innovadora por un desarrollo humano sostenible" que se realizará del 15 al 19 de febrero de 2016. En ese marco se desarrollarán el XIII Taller de Extensión Universitaria y la primera reunión de la comisión.

- XIV Congreso Latinoamericano de Extensión Universitaria tendrá como sede Managua, Nicaragua, y será en 2017.

\section{Asociación de Universidades Grupo Montevideo (AUGM)}

- Publicación de extensión: tendrá como finalidad poner en visibilidad las discusiones, políticas $y$ agendas que llevan adelante las universidades miembros del grupo en materia de extensión universitaria. La primera edición estará a cargo de la UNL.

- Reuniones anuales de la Comisión Permanente en 2016: la primera será en abril y tendrá como sede la Universidad Federal de Minas Gerais, Belo Horizonte, Brasil. La segunda será en octubre, en la Universidad de Playa Ancha, Valparaíso, Chile.

- "Il Jornadas de Extensión de AUGM": se desarrollarán en la Universidad de Playa Ancha, ciudad de Valparaíso, Chile, en el mes de octubre de 2016.

- III Congreso de Extensión Universitaria de AUGM: se realizará en 2017 en la UNL. Dicho evento girará en torno a los 100 años de la Reforma Universitaria y se enmarcará en los festejos del centenario de UNL. En ese marco se llevarán a cabo las "Primeras Jornadas de Jóvenes Extensionistas" y tendrán como principales destinatarios a estudiantes participantes de programas, proyectos $y$ prácticas de extensión.

\section{Consejo Interuniversitario \\ Nacional Red Nacional de Extensión Universitaria (REXUNI)}

- V Jornadas de Extensión del Mercosur: se realizarán los días 19 y 20 de mayo de 2016 en la Universidad Nacional del Centro (UNICEN), Tandil, Argentina. Las Jornadas son coorganizadas con la Universidad de Passo Fundo (Rio Grande do Sul, Brasil)

- VII Congreso Nacional de Extensión: se desarrollará en 2016 y tendrá como sede la Universidad Autónoma de Entre Ríos (UADER). 